\title{
Estimativa da radiação global incidente em superfícies inclinadas por modelos isotrópicos e índice de claridade
}

\author{
Adilson Pacheco de Souza(1), João Francisco Escobedo(1), Alexandre Dal Pai(2) e Eduardo Nardini Gomes ${ }^{(1)}$ \\ (1)Universidade Estadual Paulista, Faculdade de Ciências Agronômicas, Departamento de Recursos Naturais, Fazenda Experimental \\ Lageado, Rua José Barbosa de Barros, no 1.780, CEP 18610-307 Botucatu, SP. E-mail: pacheco@fca.unesp.br, escobedo@fca.unesp.br, \\ engomes@fca.unesp.br (2)Faculdade de Tecnologia de Botucatu, Departamento de Informática, Avenida José Ítalo Bacchi, s/nº, Jardim \\ Aeroporto, CEP 18606-855 Botucatu, SP. E-mail: alexandredalpai@yahoo.com.br
}

\begin{abstract}
Resumo - O objetivo deste trabalho foi avaliar o desempenho de modelos isotrópicos de estimativa do total de radiação incidente em superfícies inclinadas e propor estimativas com base nas correlações entre os índices de claridade horizontais e inclinados, em diferentes condições de cobertura de céu, em Botucatu, SP. Foram avaliadas superfícies com inclinação de $12,85^{\circ}, 22,85^{\circ}$ e $32,85^{\circ}$, pelos modelos isotrópicos propostos por Liu \& Jordan, Revfeim, Jimenez \& Castro, Koronakis, a teoria Circunsolar, e a correlação entre os índices de claridade horizontais e inclinados, para diferentes condições de cobertura de céu. O banco de dados de radiação global utilizado corresponde ao período de 1998 a 2007, com intervalos de 4/1998 a 8/2001 para a inclinação de $22,85^{\circ}$, de $9 / 2001$ a $2 / 2003$ para $12,85^{\circ}$ e de $1 / 2004$ a $12 / 2007$ para $32,85^{\circ}$. O desempenho dos modelos foi avaliado pelos indicadores estatísticos erro absoluto médio, raiz quadrada do quadrado médio do erro e índice "d" de Wilmott. Os modelos de Liu \& Jordan, Koronakis e de Revfeim apresentaram os melhores desempenhos em dias nublados, em todas as inclinações. As coberturas de céu parcialmente difuso e parcialmente aberto, nos maiores ângulos de inclinação, apresentaram as maiores dispersões entre valores estimados e medidos, independentemente do modelo. As equações estatísticas apresentaram bons resultados em aplicações com agrupamentos de dados mensais.
\end{abstract}

Termos para indexação: ângulo de inclinação, indicativos estatísticos, isotropia, radiação solar, transmissividade atmosférica.

\section{Estimation of the incident global radiation on tilted surfaces using isotropic models and clearness index}

\begin{abstract}
The objective of this work was to evaluate the performance of isotropic models estimative of the global radiation on tilted surfaces and to propose estimations based on correlation between the clearness index for horizontal and tilted surfaces, for different sky conditions, in Botucatu, SP, Brazil. The isotropic model proposed by Liu \& Jordan, Revfeim, Jimenez \& Castro, Koronakis, the Circunsolar theory and the correlation between the clearness index for horizontal and tilted surfaces, for different sky conditions, evaluated tilted surfaces at $12.85^{\circ}, 22.85^{\circ}$, and $32.85^{\circ}$. Global irradiation database used corresponds to the period from 1998 to 2007, with intervals from 4/1998 to $8 / 2001$ for the surface tilted at $22.85^{\circ}$, from $9 / 2001$ to $2 / 2003$ for the surface tilted at $12.85^{\circ}$ and from $1 / 2004$ to $12 / 2007$ for the surface tilted at $32.85^{\circ}$. The performance of the models was analyzed using the statistical indicators mean bias error, root mean square error and d-Wilmott. The models of Liu \& Jordan, Koronakis and Revfeim showed the best performance on cloudy days considering all the tilted surfaces. The sky coverage partially diffuse and partially clear with the largest tilted angles showed the highest scattering between estimated and measured values, irrespective of the model. Statistical equations showed good results in monthly average data applications.
\end{abstract}

Index terms: angle of inclination, indicative statistical, isotropy, solar irradiation, atmospheric transmission.

\section{Introdução}

A radiação solar vem se destacando nos últimos anos em pesquisas que objetivaram explorar o rendimento potencial das culturas (Lindquist et al., 2005; Amarante et al., 2007; Kunz et al., 2007; Souza et al., 2009).
Atualmente, no Brasil, encontram-se em pleno desenvolvimento tecnológico as fontes renováveis de energia, como conversão fototérmica e fotovoltaica, biomassa e biodisel, entre outras. Por conseguinte, existe uma crescente demanda por informações sobre a disponibilidade da radiação solar e a sua sazonalidade. 
Os monitoramentos e medições da radiação solar ainda são limitados, e restritos principalmente às universidades, com número reduzido de estações de superfície que registram a radiação solar, em que normalmente são realizadas medidas de apenas uma das componentes da radiação solar (radiação global), o que inviabiliza o estudo da sazonalidade das radiações, mesmo para uma única região (Soares et al., 2004; Souza et al., 2005; Tiba et al., 2005; Codato et al., 2008; Borges et al., 2010).

Estudos das intensidades e variações sazonais da radiação incidente em superfícies inclinadas por meio de séries temporais necessitam da realização de medidas por um período mínimo de cinco anos, ou então de estimativas com elevada qualidade e confiabilidade, visto que, se a radiação solar disponível em certa localidade é menor que a estimada, o desempenho dos sistemas será deficiente e as metas econômicas não serão alcançadas (Scolar et al., 2003).

Por causa da falta de medidas em superfícies inclinadas, vários modelos para sua estimativa foram desenvolvidos. Muitos desses modelos utilizam medidas das radiações global, difusa e direta em superfícies horizontais. Normalmente, nessas estimativas são empregados dois tipos de modelos: os estatísticos, ou de decomposição, e os paramétricos. Os modelos paramétricos necessitam de informações mais detalhadas das condições atmosféricas locais, conforme a componente da radiação a ser estimada, ou então a utilização de percentuais fixos de ocorrência de cada componente da radiação global (Liu \& Jordan, 1960; Notton et al., 2006; Turco \& Rizzatti, 2006; Jiang, 2009; El-Sebaii et al., 2010). Os modelos estatísticos são representados por equações matemáticas ajustadas por meio de regressão linear ou polinomial, em correlações com as frações radiométricas.

A maioria dos modelos propostos para estimativa da radiação solar foram elaborados levando-se em consideração o comportamento isotrópico da radiação (Liu \& Jordan, 1960; Revfeim, 1978; Jimenez \& Castro, 1982; Koronakis, 1986), entre outros, com enfoques principalmente na estimativa da componente difusa (Diez-Mediavilla et al., 2005; Notton et al., 2006; Loutzenhiser et al., 2007; Noorian et al., 2008), em função do seu comportamento anisotrópico em algumas condições de nebulosidade. No Brasil, os estudos mais recentes sobre o comportamento da radiação global em superfícies inclinadas e suas estimativas foram apresentados por Scolar et al. (2003) e Turco \& Rizzatti (2006).

O objetivo deste trabalho foi avaliar o desempenho de modelos isotrópicos de estimativa do total de radiação incidente em superfícies inclinadas a $12,85^{\circ}$, $22,85^{\circ}$ e $32,85^{\circ}$, e propor estimativas com base nas correlações entre os índices de claridade horizontais e inclinados, em diferentes condições de cobertura de céu, em Botucatu, SP.

\section{Material e Métodos}

Os dados de radiância solar global, direta e difusa, referentes ao período de abril de 1998 a dezembro de 2007 foram coletados na Estação de Radiometria Solar da Universidade Estadual Paulista (Unesp), em Botucatu, SP (22 $51^{\circ} \mathrm{S}, 48^{\circ} 26^{\prime \prime} \mathrm{W}$, altitude $\left.786 \mathrm{~m}\right)$. De acordo com a classificação climática de Köppen, o clima de Botucatu é Cwa, caracterizado como temperado úmido, com inverno seco e verão quente (Centro de Pesquisas Meteorológicas e Climáticas Aplicadas à Agricultura, 2009).

As leituras foram feitas a cada segundo e as médias armazenadas a cada 5 minutos, em um sistema de aquisição de dados Microlloger, modelo CR23X (Campbell Scientific, Inc., Utah, USA), na frequência de $1 \mathrm{~Hz}$. Na transferência dos dados, foi empregado o módulo de memória SM192, com interface SC532 (Campbell Scientific, Inc., Utah, USA) para microcomputadores, operado pelo software PC 208W (Campbell Scientific, Inc., Utah, USA).

As radiâncias globais no plano horizontal $\left\langle\mathrm{I}_{\mathrm{GH}}\right\rangle$ foram medidas com auxílio de piranômetros Eppley-PSP (The Eppley Laboratory, Rhode Island, USA), com fator de calibração de $7,45 \mu \mathrm{V} \mathrm{W}^{-1} \mathrm{~m}^{-2}$ e linearidade de $\pm 0,5 \%\left(0\right.$ a $\left.2.800 \mathrm{~W} \mathrm{~m}^{-2}\right)$. Para a radiância global inclinada $\left\langle\mathrm{I}_{\mathrm{G} \beta}\right\rangle$, os piranômetros foram posicionados paralelamente sobre planos inclinados de $12,45^{\circ}$ $\left\langle|\mathrm{L}|-10^{\circ}\right\rangle, 22,45^{\circ}\langle|\mathrm{L}|\rangle$ e $32,45^{\circ}\left\langle|\mathrm{L}|+10^{\circ}\right\rangle$, com a face voltada para o norte. As radiâncias diretas foram obtidas por um pireliômetro Eppley-NIP, acoplado a um rastreador solar Eppley, modelo ST3 (The Eppley Laboratory, Rhode Island, USA), com fator de calibração de 7,59 $\mu \mathrm{V} \mathrm{W}^{-1} \mathrm{~m}^{-2}$ e linearidade de $\pm 0,5 \%$ (0 a $1.400 \mathrm{~W} \mathrm{~m}^{-2}$ ). A projeção da radiância direta na horizontal foi dada pelo produto entre a radiância direta e o cosseno do ângulo zenital. A radiância difusa na horizontal foi obtida pela diferença entre a radiação global e direta. 
Os valores das radiações diária global horizontal $\left\langle\mathrm{H}_{\mathrm{GH}}^{\mathrm{d}}\right\rangle$ e inclinada $\left\langle\mathrm{H}_{\mathrm{G} \beta}^{\mathrm{d}}\right\rangle$, direta horizontal $\left\langle\mathrm{H}_{\mathrm{BH}}^{\mathrm{d}}\right\rangle$ e difusa horizontal $\left\langle\mathrm{H}_{\mathrm{DH}}^{\mathrm{d}}\right\rangle$ foram obtidos pela integração das respectivas curvas de radiância, entre o nascer e o pôr do sol (Iqbal, 1983).

$\mathrm{Na}$ inclinação de $12,45^{\circ}$, as medidas foram realizadas entre 9/2001 e 2/2003; na inclinação de $22,85^{\circ}$, entre 4/1998 e 8/2001, e na inclinação de $32,45^{\circ}$, entre $1 / 2004$ e 12/2007. Esses dados foram submetidos a uma análise de consistência, e os valores discrepantes oriundos de erros de leitura dos sensores ou mal funcionamento do sistema de aquisição de dados foram removidos das séries.

O desempenho dos seguintes modelos para estimativa da radiação global que incide em superfície inclinada com ângulo $(\beta)$ a partir de medidas na horizontal, foi analisado: Liu \& Jordan (1960), Revfeim (1978), Koronakis (1986), Jimenez \& Castro (1982), e Circunsolar (Iqbal, 1983).

A radiação diária global inclinada $\left\langle\mathrm{H}_{\mathrm{G} \beta}^{\mathrm{d}}\right\rangle$ segundo o modelo Circunsolar foi calculada pela equação (Iqbal, 1983):

$$
\left\langle\mathrm{H}_{\mathrm{G} \beta}^{\mathrm{d}}\right\rangle=\left\langle\mathrm{H}_{\mathrm{GH}}^{\mathrm{d}}\right\rangle \mathrm{R}_{\mathrm{B}},
$$

em que: $R_{B}$ é um fator geométrico decorrente da mudança no ângulo de incidência dos raios solares sobre a superfície inclinada, quando comparada com a superfície horizontal. Segundo Iqbal (1983), na partição diária, esse fator pode ser obtido diretamente por,

$\mathrm{R}_{\mathrm{B}}=\left[(\pi / 180) \omega^{\prime}{ }_{\mathrm{s}} \sin \delta \sin (\varphi-\beta)+\cos \delta \cos (\varphi-\beta) \sin \right.$ $\left.\omega_{\mathrm{s}}^{\prime}\right] /\left[(\pi / 180) \omega_{\mathrm{s}} \sin \delta \sin \varphi+\cos \delta \cos \varphi \sin \omega_{\mathrm{s}}\right]$,

em que: $\omega_{\mathrm{s}}$ é o ângulo horário solar diário horizontal, calculado pela equação:

$$
\omega_{\mathrm{s}}=\cos ^{-1}(-\tan \varphi \tan \delta),
$$

e $\omega$ 's é o ângulo horário solar diário inclinado, calculado pela equação:

$\omega_{\mathrm{S}}^{\prime}=\min \left\{\cos ^{-1}(-\tan \varphi \tan \delta) \cos ^{-1}[-\tan \delta \tan (\varphi-\beta)]\right\}$,

em que: $\varphi$ é a latitude local; $\beta$ é o ângulo de inclinação; $\delta$ é a declinação solar. Esta última é calculada pela equação:

$$
\delta=23,45 \sin [(360 / 365)(\mathrm{DJ}+284)],
$$

em que DJ é o número do dia ao longo do ano (dia juliano). A radiação diária global inclinada pelo modelo de Liu \& Jordan (1960) foi calculada pela equação:
$\left\langle\mathrm{H}_{\mathrm{G} \beta}^{\mathrm{d}}\right\rangle=\left\langle\mathrm{H}_{\mathrm{BH}}^{\mathrm{d}}\right\rangle \mathrm{R}_{\mathrm{B}}+\frac{1}{2}\left\langle\mathrm{H}_{\mathrm{DH}}^{\mathrm{d}}\right\rangle(1+\cos \beta)+\frac{1}{2} \rho\left\langle\mathrm{H}_{\mathrm{GH}}^{\mathrm{d}}\right\rangle(1-\cos \beta)$,

em que $\rho$ é o albedo da superfície.

A estimativa das radiações diária global inclinada $\left\langle\mathrm{H}_{\mathrm{G} \beta}^{\mathrm{d}}\right\rangle$ pelo modelo de Koronakis (1986) foi calculada pela equação:

$\left\langle\mathrm{H}_{\mathrm{G} \beta}^{\mathrm{d}}\right\rangle=\left\langle\mathrm{H}_{\mathrm{BH}}^{\mathrm{d}}\right\rangle \mathrm{R}_{\mathrm{B}}+\frac{1}{3}\left\langle\mathrm{H}_{\mathrm{DH}}^{\mathrm{d}}\right\rangle(2+\cos \beta)+\frac{1}{2} \rho\left\langle\mathrm{H}_{\mathrm{GH}}^{\mathrm{d}}\right\rangle(1-\cos \beta)$.

Essa estimativa se assemelha à de Liu \& Jordan (1960), todavia assume que a componente isotrópica da radiação difusa que atinge o plano inclinado equivale a $66,7 \%$ do total de radiação difusa da superfície horizontal.

O modelo de Jimenez \& Castro (1982), que estima a radiação diária global inclinada pela equação:

$$
\begin{aligned}
& \left\langle\mathrm{H}_{\mathrm{G} \beta}^{\mathrm{d}}\right\rangle=0,80\left\langle\mathrm{H}_{\mathrm{GH}}^{\mathrm{d}}\right\rangle \mathrm{R}_{\mathrm{B}}+0,2\left\langle\mathrm{H}_{\mathrm{GH}}^{\mathrm{d}}\right\rangle \frac{(1+\cos \beta)}{2} \\
& +\frac{1}{2} \rho\left\langle\mathrm{H}_{\mathrm{GH}}^{\mathrm{d}}\right\rangle(1-\cos \beta),
\end{aligned}
$$

também emprega a base teórica de Liu \& Jordan (1960), porém com a pressuposição de que a radiação difusa em um plano horizontal corresponde a $20 \%$ da radiação global.

O modelo desenvolvido por Revfeim (1978) emprega a fração radiométrica difusa e as limitações do plano de horizonte pela inclinação de uma superfície:

$$
\left.\left\langle\mathrm{H}_{\mathrm{G} \beta}^{\mathrm{d}}\right\rangle=\left\langle\mathrm{H}_{\mathrm{GH}}^{\mathrm{d}}\right\rangle\left[\mathrm{R}_{\mathrm{B}}\left(1-\mathrm{K}_{\mathrm{D}}\right)+\mathrm{f}_{\beta} \mathrm{K}_{\mathrm{D}}\right)+\rho\left(1-\mathrm{f}_{\beta}\right)\right],
$$

em que: $K_{D}$ é a razão entre a radiação difusa e a global na horizontal; $\mathrm{f}_{\beta}$ é a proporção do plano de horizonte bloqueada pela superfície inclinada, quando comparada com a superfície horizontal, estimada por $f_{\beta}=(1-\beta / 180)$. Essa fração $K_{D}$ também pode ser estimada apenas com base em medidas da radiação global na horizontal (Scolar et al., 2003).

A radiação incidente no topo da atmosfera para uma superfície horizontal $\left\langle\mathrm{I}_{0 \mathrm{H}}^{\mathrm{d}}\right\rangle$ pode ser estimada pela seguinte equação:

$$
\begin{aligned}
& \left\langle\mathrm{H}_{0 \mathrm{H}}^{\mathrm{d}}\right\rangle=\left(\frac{24}{\pi}\right) \mathrm{H}_{\mathrm{SC}} \mathrm{E}_{0}\left[\left(\frac{\pi}{180}\right) \omega_{\mathrm{s}}(\sin \delta \sin \varphi)\right. \\
& \left.+\left(\cos \delta \cos \varphi \sin \omega_{\mathrm{s}}\right)\right]
\end{aligned}
$$

em que $\mathrm{H}_{\mathrm{SC}}$ é conhecida como a constante solar, com valor igual a $4.921 \mathrm{~kJ} \mathrm{~m}^{-2} \mathrm{~h}^{-1}, \mathrm{E}_{0}$ é o fator de correção da excentricidade da órbita da Terra. Segundo Iqbal (1983), o fator de excentricidade pode ser calculado por: 
$\mathrm{E}_{0}=1,000110+0,034221 \cos \Gamma+0,001280 \sin \Gamma+0,000719$ $\cos 2 \Gamma+0,000077 \sin 2 \Gamma$, em que $\Gamma=2 \pi(\mathrm{DJ}-1) / 366$.

Além desses modelos de estimativa, foram propostas neste trabalho correlações entre as frações da radiação global inclinada $\left\langle\mathrm{K}_{\mathrm{T} \beta}^{\mathrm{d}}\right\rangle$. Os índices $\left\langle\mathrm{K}_{\mathrm{T} \beta}^{\mathrm{d}}\right\rangle$ são obtidos pela razão entre a radiação global e a radiação no topo da atmosfera $\left\langle\mathrm{H}_{0 \beta}^{\mathrm{d}}\right\rangle$ para superfícies inclinadas:

$$
\begin{aligned}
& \left\langle\mathrm{H}_{0 \beta}^{\mathrm{d}}\right\rangle=\left(\frac{24}{\pi}\right) \mathrm{I}_{\mathrm{SC}} \mathrm{E}_{0}\left\{\left(\frac{\pi}{180}\right) \omega_{\mathrm{s}}[\sin \delta \sin (\varphi-\beta)]\right. \\
& \left.+\left(\cos \delta \cos (\varphi-\beta) \sin \omega_{\mathrm{s}}^{\prime}\right)\right\} .
\end{aligned}
$$

Nas correlações entre os valores estimados pelos modelos de estimativa da radiação global inclinada supracitados e os valores medidos de $\left\langle\mathrm{H}_{\mathrm{G} \beta}^{\mathrm{d}}\right\rangle$, foram usados dados de janeiro a dezembro de 2002, para a inclinação de $12,85^{\circ}$, de janeiro de 1999 a dezembro de 2000 , para $22,85^{\circ}$, e de janeiro de 2004 a dezembro de 2005 para $32,85^{\circ}$. Foram empregados, nas análises de desempenho das estimativas, dados dos seguintes períodos: de abril de 1998 a dezembro de 1998 e de janeiro a agosto de 2001, para a inclinação $22,85^{\circ}$; de setembro a dezembro de 2001 e de janeiro a fevereiro de 2003, para a inclinação $12,85^{\circ}$; e de janeiro a dezembro de 2006, para a inclinação $32,85^{\circ}$.

$\mathrm{Na}$ avaliação do desempenho das equações de estimativa diária dos seis modelos e das correlações propostas, foram empregados os indicadores estatísticos erro absoluto médio ("Mean bias error" $\mathrm{MBE}$ ), raiz quadrada do quadrado médio do erro ("Root mean square error" RMSE), recomendados por Iqbal (1983), e o índice de ajustamento "d" de Willmott, para diferentes condições de coberturas de céu (Diez-Mediavilla et al., 2005; Notton et al., 2006; Loutzenhiser et al., 2007; Escobedo et al., 2009; Evseev \& Kudish, 2009; El-Sebaii et al., 2010). Esses indicadores foram calculados pelas seguintes equações:

$$
\begin{aligned}
& \text { MBE }=\sum_{i=1}^{N}(\mathrm{Pi}-\mathrm{Oi}) / \mathrm{N} ; \\
& \operatorname{RMSE}=\left[\sum_{\mathrm{i}=1}^{\mathrm{N}}(\mathrm{Pi}-\mathrm{Oi}) / \mathrm{N}\right]^{0,5} \mathrm{e}, \\
& \mathrm{d}=1-\left[\sum_{\mathrm{i}=1}^{\mathrm{N}}(\mathrm{Pi}-\mathrm{Oi})^{2}\right] /\left[\sum_{\mathrm{i}=1}^{\mathrm{N}}\left(\left|\mathrm{P}^{\prime} \mathrm{i}\right|+\left|\mathrm{O}^{\prime} \mathrm{i}\right|\right)^{2}\right] ;
\end{aligned}
$$

em que: Pi representa os valores estimados, Oi os valores medidos, $\mathrm{N}$ é o número de observações, $\left|\mathrm{P}^{\prime} \mathrm{i}\right|$ é o valor absoluto da diferença $\mathrm{Pi}$ - $\overline{\mathrm{O}}$, e $\left|\mathrm{O}^{\prime} \mathrm{i}\right|$ representa o valor absoluto da diferença Oi - Ōi.

A classificação da cobertura de céu em função do índice de claridade $\left\langle\mathrm{K}_{\mathrm{BH}}^{\mathrm{d}}\right\rangle$ empregada neste trabalho seguiu a proposta de Escobedo et al. (2009), dada pela seguinte classificação de cobertura de céu para a partição diária: $\left\langle\mathrm{K}_{\mathrm{T}}^{\mathrm{d}}\right\rangle \leq 0,35$, a radiação direta é praticamente nula, sendo a radiação global igual à radiação difusa (céu nublado); $0,35 \leq\left\langle\mathrm{K}_{\mathrm{T}}^{\mathrm{d}}\right\rangle \geq 0,55$, a radiação global é composta principalmente pela fração difusa e em menor escala pela radiação direta (céu parcialmente difuso); $0,55 \leq\left\langle\mathrm{K}_{\mathrm{T}}^{\mathrm{d}}\right\rangle \leq 0,65$, é o inverso do segundo intervalo, visto que a radiação difusa diminui gradativamente (céu parcialmente aberto); $\left\langle\mathrm{K}_{\mathrm{T}}^{\mathrm{d}}\right\rangle>0,65$, a radiação global possui a maior parcela composta pela radiação direta e mínima difusa, portanto, a cobertura do céu é considerada aberta. Para valores de $\left\langle\mathrm{K}_{\mathrm{T}}^{\mathrm{d}}\right\rangle$ acima de 0,78 , não ocorre uma sequência lógica de evolução das três radiações em função da transmissividade atmosférica e, por essa razão, foram eliminados das análises e também dos modelos de estimativas gerados.

\section{Resultados e Discussão}

Em relação à frequência de distribuição dos valores de $\left\langle\mathrm{K}_{\mathrm{TH}}^{\mathrm{d}}\right\rangle$ em diferentes condições de cobertura de céu, observou-se que, no município de Botucatu, SP, foi verificado no máximo 70 dias de céu nublado - valores de $\left\langle\mathrm{K}_{\mathrm{TH}}^{\mathrm{d}}\right\rangle$ inferiores a 0,35 - em 2004, o que corresponde a $19,18 \%$ dos dias ao longo do ano (Figura 1). Em torno de $65,48,58,08,61,37,52,52$ e $55,07 \%$ dos dias dos anos de 1999, 2000, 2002, 2004 e 2005, respectivamente, apresentaram índices de claridade acima de 0,55, indicando que na maioria dos dias a atmosfera local transmite acima de $55 \%$ da radiação incidente no topo da atmosfera para o plano horizontal. Escobedo et al. (2009) verificaram, nesse mesmo município, que, entre janeiro de 2001 e dezembro de 2004, ocorreram 217 dias nublados, 215 dias com céu parcialmente difuso, 271 dias com céu parcialmente aberto e 490 dias com céu aberto.

Os modelos isotrópicos Liu \& Jordan (1960), Revfeim (1978), Jimenez \& Castro (1982), Koronakis (1986) apresentaram ótimas correlações entre os valores medidos e estimados de radiação global 
incidente, nas três inclinações estudadas (Figura 2). Os coeficientes de determinação nas correlações desses modelos foram em torno de 0,98 , com tendência de diminuição dos valores de $\mathrm{R}^{2}$ com o incremento do ângulo de inclinação, como pode ser evidenciado para o modelo Jimenez \& Castro (1982), que apresentou valores de $\mathrm{R}^{2}$ de 0,$99 ; 0,98$ e 0,97 , para as inclinações de $12,85^{\circ}, 22,85^{\circ}$ e $32,85^{\circ}$, respectivamente.

A estimativa de $\left\langle\mathrm{H}_{\mathrm{G} \beta}^{\mathrm{d}}\right\rangle$ com base na teoria circunsolar apresentou correlações semelhantes aos modelos isotrópicos, para pequenas inclinações (Figura 2). Todavia, com o aumento do ângulo de inclinação, a dispersão gráfica dos dados aumentou significativamente, possibilitando menores valores do coeficiente de determinação.

Nos menores ângulos de inclinação $\left(12,85^{\circ}\right)$, não ocorreu variação no comportamento dos modelos isotrópicos nas diferentes coberturas de céu (Figura 2). Porém, em ângulos maiores $\left(32,85^{\circ}\right)$, as maiores dispersões foram originadas em dias com céu parcialmente aberto $\left(0,55<\left\langle\mathrm{K}_{\mathrm{TH}}^{\mathrm{d}}\right\rangle>0,65\right)$ e aberto $\left(\left\langle\mathrm{K}_{\mathrm{TH}}^{\mathrm{d}}\right\rangle>0,65\right)$. Para estimativas com o modelo Circunsolar, as maiores dispersões ocorreram em dias com cobertura de céu parcialmente nublado, tanto difuso quanto aberto.

Em dias com céu aberto, a disponibilidade de densidade de fluxo de radiação solar é maior, normalmente superior a $15 \mathrm{MJ} \mathrm{m}^{-2} \mathrm{dia}^{-1}$, decorrente do aumento das componentes direta e refletida, em contrapartida da diminuição da radiação difusa. As

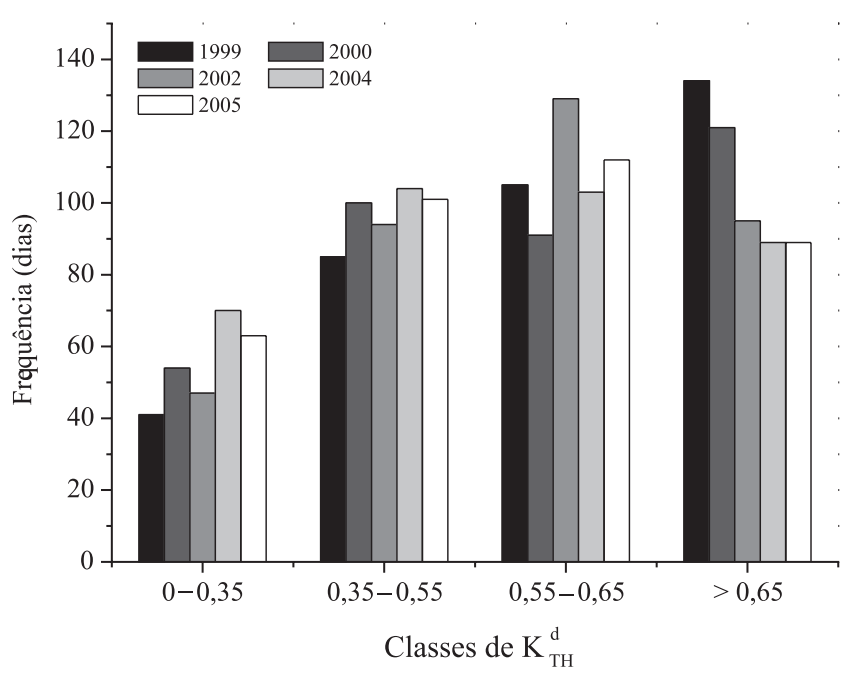

Figura 1. Freqüência de distribuição dos valores diários do índice de claridade $\left\langle\mathrm{K}_{\mathrm{TH}}^{\mathrm{d}}\right\rangle$ ao longo do ano para Botucatu, SP. maiores dispersões para céu aberto podem ser explicadas pelo aumento da componente refletida, decorrentes do termo $\left[\frac{1}{2} \rho\left\langle\mathrm{H}_{\mathrm{GH}}^{\mathrm{d}}\right\rangle(1-\cos \beta)\right]$, ou seja, é grandemente dependente dos maiores níveis de $\left\langle\mathrm{H}_{\mathrm{GH}}^{\mathrm{d}}\right\rangle$. Essas dispersões ainda são decorrentes da forma com que a radiação difusa é originada nesses dias (Iqbal, 1983; Gueymard, 2009). Scolar et al. (2003) verificaram que modelos isotrópicos de estimativa de $\left\langle\mathrm{H}_{\mathrm{G} \beta}^{\mathrm{d}}\right\rangle$ apresentam correções da componente difusa com bons resultados, em dias com céu nublado, pois desconsideram o comportamento anisotrópico de dias com céu aberto.

Contudo, o modelo Circunsolar apresentou menores dispersões em dias de céu aberto do que em dias de céu nublado ou parcialmente difuso, em consequência de a maior percentagem da radiação difusa em dias de céu aberto ser proveniente do disco solar, ou seja, a componente circunsolar (Iqbal, 1983; Scolar et al., 2003).

Em relação aos desempenhos dos cinco modelos isotrópicos de estimativa, nos agrupamentos em diferentes condições de cobertura de céu e com todos os dados, observou-se que, independentemente das classes de $\left\langle\mathrm{K}_{\mathrm{TH}}^{\mathrm{d}}\right\rangle$, todos os modelos apresentaram tendência de subestimar os valores de $\left\langle\mathrm{H}_{\mathrm{G} \beta}^{\mathrm{d}}\right\rangle$ para a inclinação de $12,85^{\circ}$ (Figura 3).

Para os agrupamentos com todos os dados, em todas as inclinações estudadas, o modelo Revfeim (1978) subestimou os valores de $\left\langle\mathrm{H}_{\mathrm{G} \beta}^{\mathrm{d}}\right\rangle$. Ainda nesse agrupamento de dados, as maiores superestimativas foram obtidas pelo modelo Circunsolar em 32,85 $\left(0,347 \mathrm{MJ} \mathrm{m}^{-2} \mathrm{dia}^{-1}\right)$, que corresponderam em termos percentuais de desvios entre os valores médios mensais medidose estimados em-5,15e 1,93\%, respectivamente. Nas diferentes condições de cobertura de céu, o modelo Revfeim (1978) superestimou os valores de $\left\langle\mathrm{H}_{\mathrm{G} \beta}^{\mathrm{d}}\right\rangle$ apenas em céu aberto, para a inclinação de $32,85^{\circ}$ $\left(0,329 \mathrm{MJ} \mathrm{m}^{-2} \mathrm{dia}^{-1}\right.$ ou 1,37\%). Todos os modelos apresentaram tendências de superestimativas e subestimativas similares, em todas as inclinações e coberturas de céu, que variaram entre $-0,621 \mathrm{MJ} \mathrm{m}^{-2}$ $\operatorname{dia}^{-1}$ ou $-3,78 \%$, no modelo Revfeim (1978), em $\left\langle|\mathrm{L}|+10^{\circ}\right\rangle$ para céu parcialmente difuso, e $0,510 \mathrm{MJ} \mathrm{m}^{-2}$ $\operatorname{dia}^{-1}$ ou $6,61 \%$, no modelo Jimenez \& Castro (1982), em $\left\langle|\mathrm{L}|+10^{\circ}\right\rangle$ para céu nublado.

As maiores dispersões entre valores estimados e medidos de $\left\langle\mathrm{H}_{\mathrm{G} \beta}^{\mathrm{d}}\right\rangle$ foram verificadas na aplicação do modelo Circunsolar, sendo crescente com o aumento 

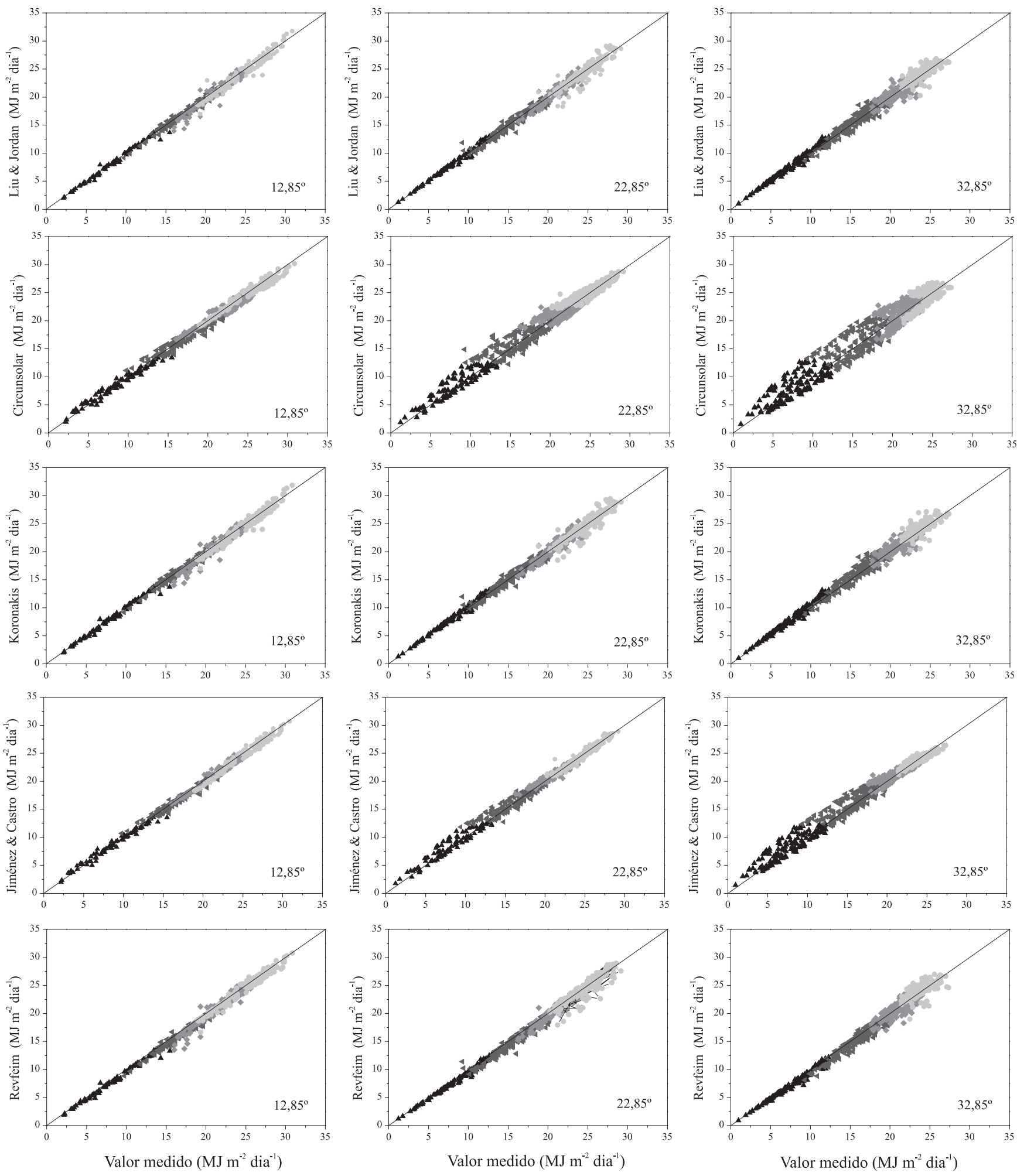

$\Delta$ Nublado $\triangleleft$ Parcialmente nublado difuso $\diamond$ Parcialmente nublado $\curvearrowright$ Aberto

Figura 2. Correlações entre valores medidos e estimados pelos modelos Liu \& Jordan (1960), Circunsolar (Iqbal, 1983), Koronakis (1986), Jimenez \& Castro (1982) e Revfeim (1978) de radiação global incidente em superfícies inclinadas a $12,85^{\circ}, 22,85^{\circ}$ e $32,85^{\circ}$, com a face voltada para o Norte, em diferentes condições de cobertura do céu, em Botucatu, SP. 
do índice de claridade e equivalentes a $0,762,1,606$ e $2,012 \mathrm{MJ} \mathrm{m}^{-2}$ dia $^{-1}$ para as inclinações de $12,85,22,85$ e $32,85^{\circ}$, em dias com céu parcialmente difuso, respectivamente. Nos cinco modelos isotrópicos estudados, os índices de ajustamento apresentaram diminuição com o aumento da inclinação e diminuição da nebulosidade. Com exceção do modelo Circunsolar, os ajustamentos permaneceram superiores a $90 \%$ em todas as condições de cobertura de céu. Essas variações nos valores de MBE, RMSE e "d" são verificadas na maioria dos trabalhos na partição diária da radiação global (Diez-Mediavilla et al., 2005; Notton et al., 2006; Loutzenhiser et al., 2007; Escobedo et al., 2009; Evseev \& Kudish, 2009).

Quanto às estimativas de $\left\langle\mathrm{H}_{\mathrm{G} \beta}^{\mathrm{d}}\right\rangle$, com base nas correlações entre $\left\langle\mathrm{K}_{\mathrm{T} \beta}^{\mathrm{d}}\right\rangle$ e $\left\langle\mathrm{K}_{\mathrm{TH}}^{\mathrm{d}}\right\rangle$, observou-se que as medidas de $\left\langle\mathrm{K}_{\mathrm{G} \beta}^{\mathrm{d}}\right\rangle$ nos ângulos de 12,85 e $32,85^{\circ}$ foram maiores do que as verificadas no plano horizontal, em função dos coeficientes angulares serem superiores a 1 (Figura 4). Na inclinação de $22,85^{\circ}$, ocorreu uma inversão, com indicação de que a radiação global na horizontal é maior do que na inclinada. Nota-se uma linearidade entre os valores de $\left\langle\mathrm{K}_{\mathrm{T} \beta}^{\mathrm{d}}\right\rangle \mathrm{e}\left\langle\mathrm{K}_{\mathrm{TH}}^{\mathrm{d}}\right\rangle$, visto que para pequenos valores de $\left\langle\mathrm{K}_{\mathrm{TH}}^{\mathrm{d}}\right\rangle$ são encontrados pequenos valores de $\left\langle\mathrm{K}_{\mathrm{T} \beta}^{\mathrm{d}}\right\rangle$.

No desempenho das estimativas da radiação global, em superfícies inclinadas com base na radiação global na horizontal, foram encontrados coeficientes de determinação $\left(\mathrm{R}^{2}\right)$ acima de 0,88 , que em conjunto com os demais índices estatísticos permitiram boas estimativas. Essas correlações apresentaram tendências a superestimar os valores de $\left\langle\mathrm{K}_{\mathrm{T} \beta}^{\mathrm{d}}\right\rangle$, com MBE de 1,98, 5,36 e $6,93 \%$, e índices de ajustamento em torno de 0,99 . Todavia, quando os modelos são validados em função das frações estimadas, não são possíveis análises em termos de energia. As três equações ajustadas

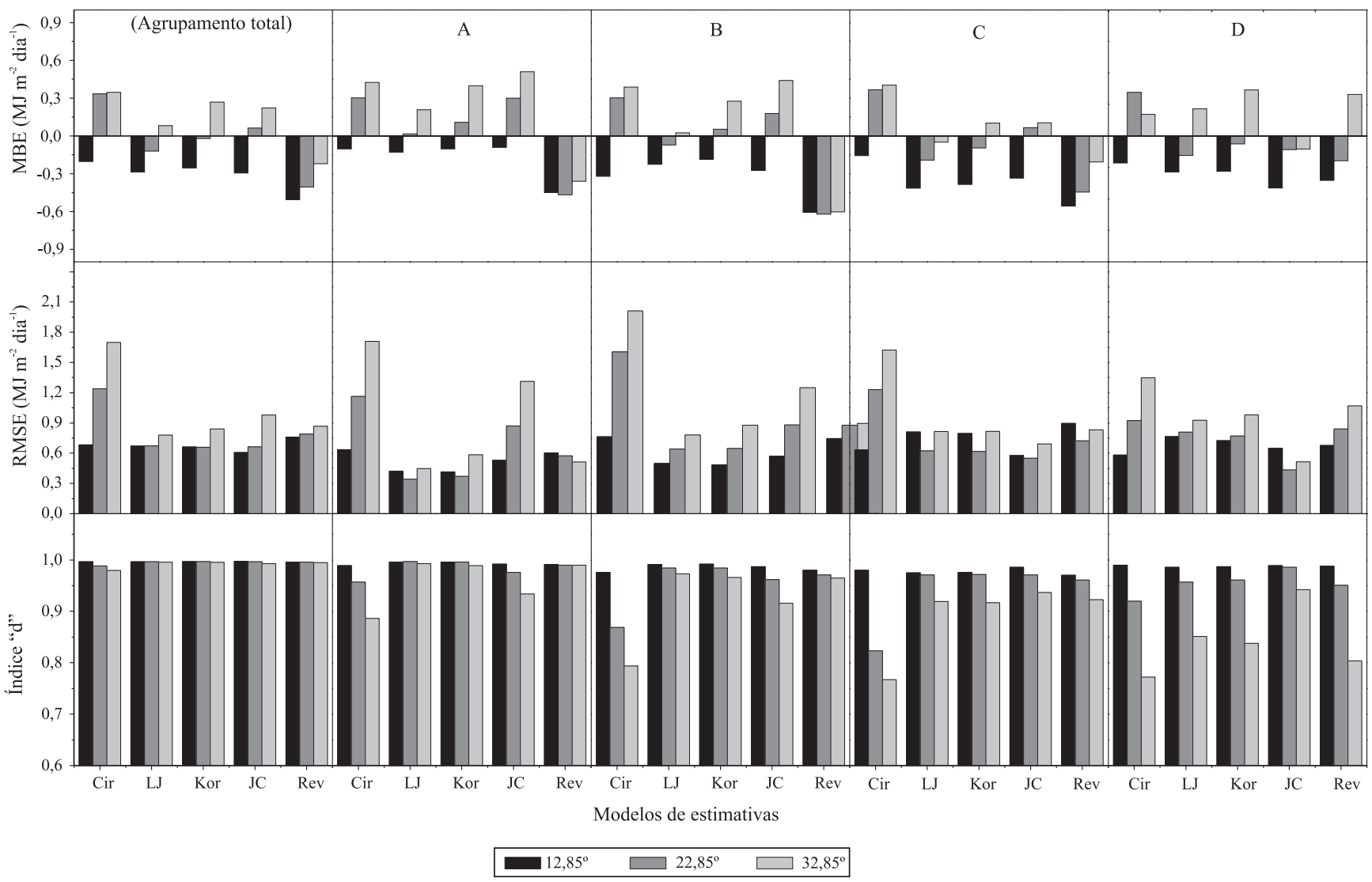

Figura 3. Indicativos estatísticos (MBE, erro absoluto médio; RMSE, raiz quadrada do quadrado médio do erro e índice de ajustamento "d" de Willmott) dos modelos: Circunsolar (Iqbal, 1983) - (Cir); Liu \& Jordan (1960) - (LJ); Koronakis (1986) - (Kor); Jimenez \& Castro (1982) - (JC) e Revfeim (1978) - (Rev) de estimativa da radiação global incidente em superfícies inclinadas a $12,85^{\circ}, 22,85^{\circ}$ e $32,85^{\circ}$, com a face voltada para o Norte, em dias com céu nublado (A), céu parcialmente nublado difuso (B), céu parcialmente nublado aberto (C) e céu aberto (D), em Botucatu, SP. 
apresentaram tendência de superestimar os valores de $\left\langle\mathrm{H}_{\mathrm{G} \beta}^{\mathrm{d}}\right\rangle$, com valores de MBE crescentes com o aumento do ângulo de inclinação. Em 12,85, 22,85 e 32,85 ${ }^{\circ}$, o MBE foi de $0,394 \mathrm{MJ} \mathrm{m}^{-2}(1,93 \%), 1,025 \mathrm{MJ} \mathrm{m}^{-2}$ $(5,30 \%)$ e $1,485 \mathrm{MJ} \mathrm{m}^{-2}$ (7,86\%), respectivamente. As dispersões entre valores estimados e medidos de $\left\langle\mathrm{H}_{\mathrm{G} \beta}^{\mathrm{d}}\right\rangle$ também seguiram essa tendência de aumento com a intensidade de inclinação, e variaram de $1,186 \mathrm{MJ} \mathrm{m}^{-2}$ $(5,81 \%)$ a $3,733 \mathrm{MJ} \mathrm{m}^{-2}(19,76 \%)$. Consequentemente,
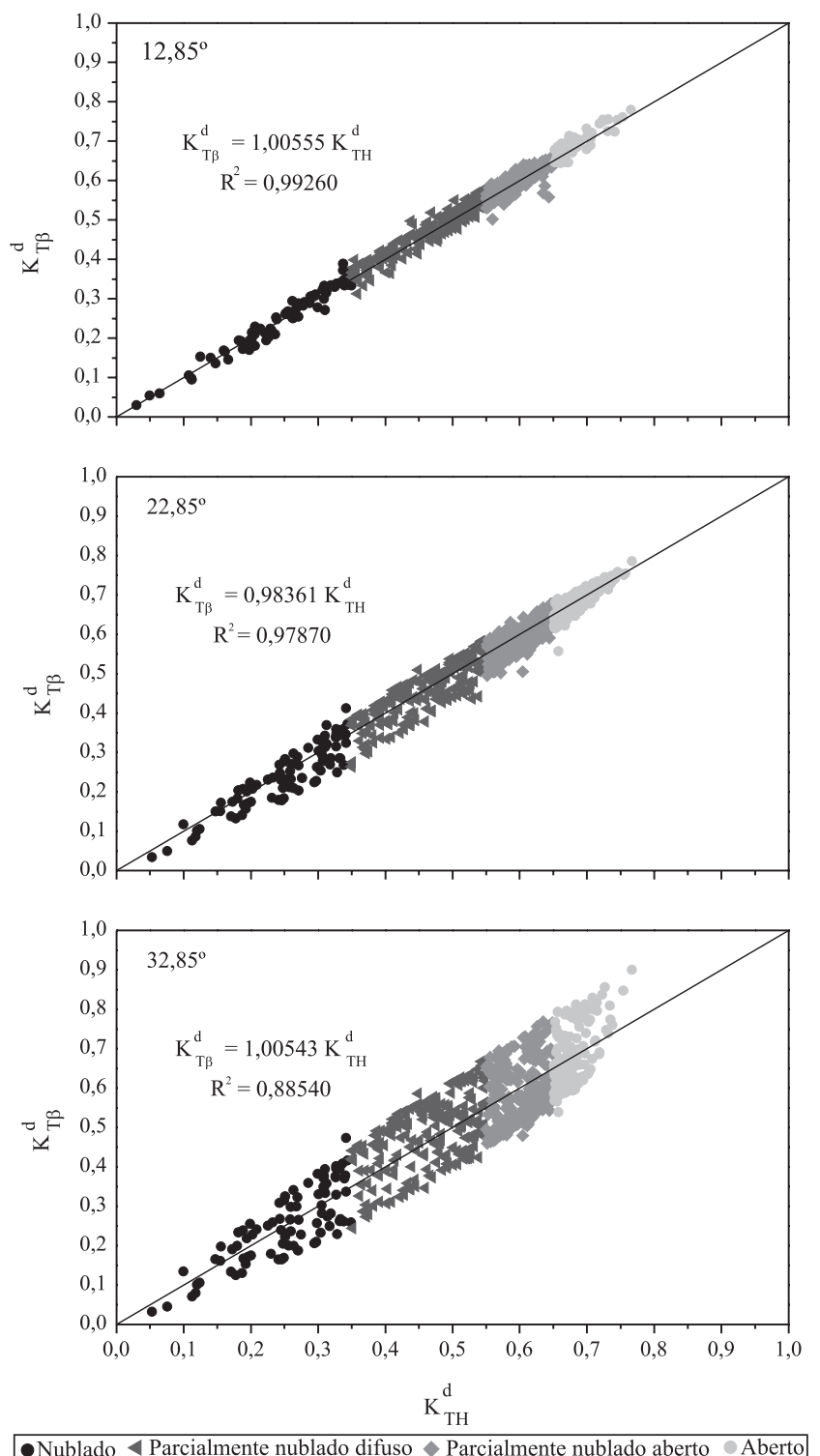

Figura 4. Correlações entre radiação global inclinada $\left\langle\mathrm{K}_{\mathrm{T} \beta}^{\mathrm{d}}\right\rangle \mathrm{e}$ índice de claridade $\left\langle\mathrm{K}_{\mathrm{TH}}^{\mathrm{d}}\right\rangle$ para estimativa da radiação global incidente em superfícies inclinadas a $12,85^{\circ}, 22,85^{\circ}$ e $32,85^{\circ}$, com a face voltada para o Norte, em diferentes condições de cobertura do céu em Botucatu, SP. os índices de ajustamento foram reduzidos em função das inclinações, sendo de 0,9901, 0,95227 e 0,87918, para $12,85,22,85$ e $32,85^{\circ}$, respectivamente. As maiores dispersões verificadas com o aumento da inclinação foram decorrentes do aumento dos níveis da radiação refletida em superfícies inclinadas com maiores ângulos e também do ganho de radiação direta nas maiores inclinações, no período de inverno.

Os valores médios mensais medidos e estimados de $\left\langle\mathrm{H}_{\mathrm{G} \beta}^{\mathrm{d}}\right\rangle$ correspondem aos mesmos períodos de medidas na avaliação do desempenho das estimativas com correlações entre $\left\langle\mathrm{K}_{\mathrm{T} \beta}^{\mathrm{d}}\right\rangle \mathrm{e}\left\langle\mathrm{K}_{\mathrm{TH}}^{\mathrm{d}}\right\rangle$. Observou-se que, para a estimativa na inclinação de $12,85^{\circ}$ nos meses de janeiro, fevereiro, novembro e dezembro, todos os modelos subestimaram os valores de $\left\langle\mathrm{H}_{\mathrm{G} \beta}^{\mathrm{d}}\right\rangle$, sendo os valores medidos superiores em até $24,14 \%$, em novembro, para o modelo Liu \& Jordan (1960) (Figura 5). Ainda para essa inclinação, o modelo Jimenez \& Castro (1982) apresentou melhores resultados, em estimativas no mês janeiro $(-2,66 \%)$, fevereiro $(-0,94 \%)$ e novembro $(-0,68 \%)$, enquanto o modelo Revfeim (1978) foi melhor em outubro $(+0,67 \%)$ e dezembro (-0,98\%), e o modelo Circunsolar foi superior em setembro $\left(+0,02 \mathrm{MJ} \mathrm{m}^{-2} \mathrm{dia}^{-1}\right)$.

Para a inclinação de $22,85^{\circ}$, os modelos Liu \& Jordan (1960) e Revfeim (1978) subestimaram, enquanto o modelo Revfeim (1978) superestimou, em todos os meses, os valores de $\left\langle\mathrm{H}_{\mathrm{G} \beta}^{\mathrm{d}}\right\rangle$ (Figura 5). Os modelos gerados pelas correlações entre os índices de claridade horizontal $\left\langle\mathrm{H}_{\mathrm{TH}}^{\mathrm{d}}\right\rangle$ e inclinados $\left\langle\mathrm{H}_{\mathrm{T} \beta}^{\mathrm{d}}\right\rangle$ e Circunsolar apresentaram tendências de superestimar os valores de $\left\langle\mathrm{H}_{\mathrm{G} \beta}^{\mathrm{d}}\right\rangle$ no verão e subestimar nos meses de inverno. As maiores subestimativas foram de $27,49 \%$, ou $5,42 \mathrm{MJ} \mathrm{m}^{-2} \mathrm{dia}^{-1}$, em fevereiro, para o modelo Liu \& Jordan (1960), e as maiores superestimavas foram propiciadas pelo método das correlações entre os índices de claridade horizontal e inclinados no mês de junho ( $+9,33 \%$, ou $\left.1,67 \mathrm{MJ} \mathrm{m}^{-2} \mathrm{dia}^{-1}\right)$. Por conseguinte, para estimativas na inclinação de $32,85^{\circ}$, os modelos Koronakis (1986) e Liu \& Jordan (1960) apresentaram comportamentos semelhantes, superestimando os valores de $\left\langle\mathrm{H}_{\mathrm{G} \beta}^{\mathrm{d}}\right\rangle$ nos meses de novembro e dezembro em, no máximo, $0,91 \mathrm{MJ} \mathrm{m}^{-2} \mathrm{dia}^{-1}$. Os piores resultados foram verificados pelos modelos das correlações entre os índices de claridade horizontal e inclinados, nos meses de inverno, com superestimativas de até $23,09 \%(4,48$ $\mathrm{MJ} \mathrm{m}^{-2}$ dia $\left.^{-1}\right)$ no mês de julho.

De Miguel et al. (1995) verificaram que os modelos Koronakis (1986) e Liu \& Jordan (1960) apresentaram 

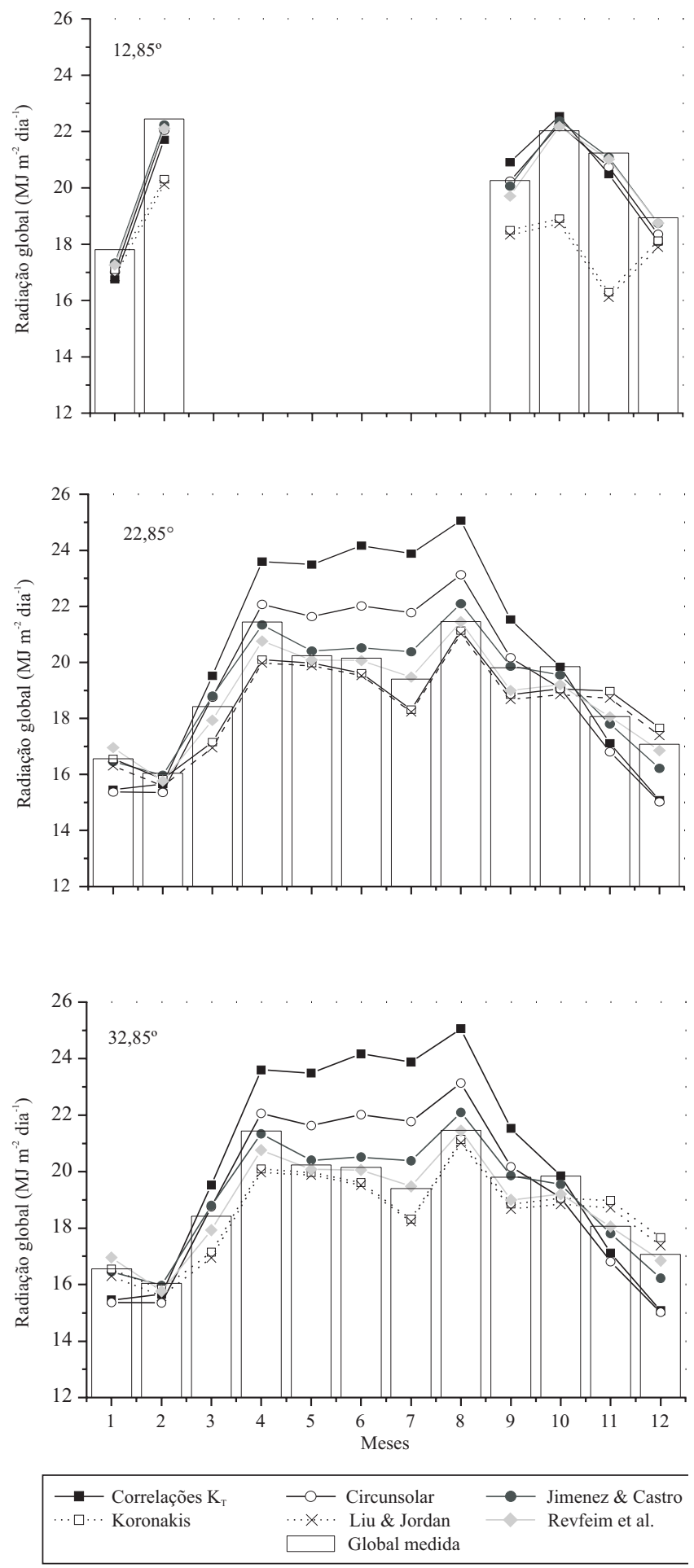

Figura 5. Valores médios mensais da radiação global inclinada medida e estimada pelos modelos isotrópicos Liu \& Jordan (1960), Revfeim (1978), Jimenez \& Castro (1982), Circunsolar (Iqbal, 1983) e Koronakis (1986) para superfícies inclinadas a $12,85^{\circ}, 22,85^{\circ}$ e $32,85^{\circ}$, com a face voltada para o Norte, em Botucatu, SP. Correlações $\mathrm{K}_{\mathrm{T}}$ referem-se a correlações entre índice de claridade. resultados similares na estimativa de $\left\langle\mathrm{H}_{\mathrm{G} \beta}^{\mathrm{d}}\right\rangle$ em Burgos, na Espanha, região localizada na latitude $42^{\circ} 20^{\prime} 25^{\prime \prime}$, para superfícies inclinadas até $40^{\circ}$, com face voltada para o sul. Entre os modelos Koronakis (1986) e Jimenez \& Castro (1982), esses mesmos autores verificaram similaridades nas estimativas apenas nos meses de verão. Tian et al. (2001) concluíram que o modelo Revfeim (1978) estimou satisfatoriamente os valores de $\left\langle\mathrm{H}_{\mathrm{G} \beta}^{\mathrm{d}}\right\rangle$, considerando as variações de cobertura de céu para as cidades de Kataia e Invercargill (latitudes $35^{\circ}$ e $46^{\circ} \mathrm{S}$ ), na Nova Zelândia.

Kamali et al. (2006) estudaram oito modelos de estimativa de $\left\langle\mathrm{H}_{\mathrm{G} \beta}^{\mathrm{d}}\right\rangle$ para superfícies inclinadas a $45^{\circ} \mathrm{e}$ $40^{\circ}$, com faces voltadas para o sul e leste, respectivamente, em Karaj, Irã (latitude $35^{\circ} 55^{\prime} \mathrm{N}$ ) e verificaram que o modelo Koronakis (1986) apresentou os melhores desempenhos na predição da componente difusa para a face leste.

Para melhorar o desempenho das equações geradas pelas correlações entre $\left\langle\mathrm{K}_{\mathrm{T} \beta}^{\mathrm{d}}\right\rangle \mathrm{e}\left\langle\mathrm{K}_{\mathrm{TH}}^{\mathrm{d}}\right\rangle$, foram propostas equações geradas com agrupamentos mensais (Tabela 1), dadas por regressão linear simples, como $\mathrm{K}_{\mathrm{T} \beta}^{\mathrm{d}}=\mathrm{a}\left\langle\mathrm{K}_{\mathrm{TH}}^{\mathrm{d}}\right\rangle$. Neste caso, existem dois períodos distintos que acompanham as evoluções das radiações global e extraterrestre. No verão, as superfícies horizontais apresentam níveis incidentes maiores que os verificados na inclinada, com coeficientes angulares maiores que 1,0 e, entre os equinócios, essa situação se inverte. As piores correlações foram verificadas no mês de abril, dadas pelo início da entrada das frentes frias na região, o que propicia uma grande variação no número de dias com céu nublado e céu aberto. Nos meses de verão, dezembro e janeiro, as equações para as inclinações de 22,85 e $32,85^{\circ}$ apresentaram tendências de subestimar, dadas pelos valores de MBE negativos (Tabela 2).

$\mathrm{Na}$ inclinação de $32,85^{\circ}$, foram verificados os maiores desvios das médias nas estimativas de $\left\langle\mathrm{H}^{\mathrm{d}}{ }_{\mathrm{G} \beta}\right\rangle$, com equações mensais de inverno apresentando variação entre 1,30 e $1,90 \mathrm{MJ} \mathrm{m}^{-2}$ entre os meses de maio e agosto. Acima de $85 \%$ dos modelos mensais superestimam os valores de $\left\langle\mathrm{H}_{\mathrm{G} \beta}^{\mathrm{d}}\right\rangle$. Para menores períodos no agrupamento de dados, foram verificados melhores ajustamentos em todas as inclinações. Todavia, nos meses em que ocorre maior número de dias com céu parcialmente nublado, os modelos mensais apresentaram os piores desempenhos, para os três indicativos analisados. 
Tabela 1. Coeficiente angular (a1) e de determinação (R2) das equações lineares $\mathrm{K}_{\mathrm{Tb}}^{\mathrm{d}}=\mathrm{a}\left\langle\mathrm{K}_{\mathrm{TH}}^{\mathrm{d}}\right\rangle$ mensais, para estimativa da radiação global em superfícies inclinadas com base na horizontal.

\begin{tabular}{|c|c|c|c|c|c|c|}
\hline \multirow[t]{2}{*}{ Mês } & \multicolumn{2}{|c|}{$12,85^{\circ}$} & \multicolumn{2}{|c|}{$22,85^{\circ}$} & \multicolumn{2}{|c|}{$32,85^{\circ}$} \\
\hline & $a_{1}$ & $\mathrm{R}^{2}$ & $a_{1}$ & $\mathrm{R}^{2}$ & $a_{1}$ & $\mathrm{R}^{2}$ \\
\hline Janeiro & 1,0363 & 0,9966 & 1,0463 & 0,9961 & 1,0947 & 0,9944 \\
\hline Fevereiro & 1,0068 & 0,9969 & 1,0252 & 0,9979 & 1,0485 & 0,9964 \\
\hline Março & 0,9763 & 0,9935 & 0,9929 & 0,9924 & 0,9965 & 0,9946 \\
\hline Abril & 0,9795 & 0,9283 & 0,9743 & 0,9987 & 0,9463 & 0,9880 \\
\hline Maio & 0,9820 & 0,9981 & 0,9443 & 0,9914 & 0,9235 & 0,9949 \\
\hline Junho & 0,9866 & 0,9888 & 0,9311 & 0,9936 & 0,9008 & 0,9924 \\
\hline Julho & 0,9871 & 0,9968 & 0,9365 & 0,9902 & 0,9089 & 0,9942 \\
\hline Agosto & 0,9909 & 0,9986 & 0,8927 & 0,9006 & 0,9560 & 0,9941 \\
\hline Setembro & 0,9975 & 0,9985 & 0,9721 & 0,9946 & 0,9828 & 0,9957 \\
\hline Outubro & 1,0081 & 0,9944 & 1,0075 & 0,9984 & 1,0239 & 0,9927 \\
\hline Novembro & 1,0289 & 0,9978 & 1,0298 & 0,9981 & 1,0624 & 0,9957 \\
\hline Dezembro & 1,0361 & 0,9978 & 1,0477 & 0,9980 & 1,0928 & 0,9953 \\
\hline
\end{tabular}

Tabela 2. Avaliação de desempenho( ${ }^{(1)}$ das relações entre radiação global inclinada e índice de claridade com agrupamentos mensais e equações mensais.

\begin{tabular}{|c|c|c|c|c|c|c|c|}
\hline \multirow[t]{2}{*}{ Mês } & \multicolumn{2}{|c|}{ Valor medido $\quad$ Valor estimado } & MBE & RMSE & MBE & RMSE & \multirow[t]{2}{*}{$\mathrm{d}$} \\
\hline & \multicolumn{2}{|c|}{ 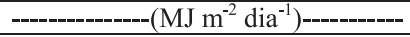 } & \multicolumn{2}{|c|}{ 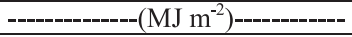 } & \multicolumn{2}{|c|}{ 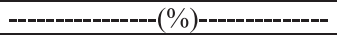 } & \\
\hline & \multicolumn{4}{|l|}{ Inclinação $12,85^{\circ}$} & & & \\
\hline Janeiro & 17,811 & 17,828 & 0,017 & 0,596 & 0,093 & 3,347 & 0,9974 \\
\hline Fevereiro & 22,449 & 22,529 & $0,079^{(2)}$ & 1,096 & $0,353^{(3)}$ & 4,881 & 0,9905 \\
\hline Setembro & 20,263 & 21,741 & 1,478 & 1,511 & 7,293 & 7,456 & 0,9811 \\
\hline Outubro & 22,030 & 23,558 & $1,529^{(2)}$ & $1,883^{(2)}$ & $6,938^{(3)}$ & $8,545^{(3)}$ & $0,9701^{(4)}$ \\
\hline Novembro & 21,233 & 21,747 & $0,514^{(2)}$ & $0,620^{(2)}$ & $2,420^{(3)}$ & $2,920^{(3)}$ & $0,9962^{(4)}$ \\
\hline \multirow[t]{2}{*}{ Dezembro } & 18,935 & 19,239 & $0,304^{(2)}$ & $0,732^{(2)}$ & $1,605^{(3)}$ & $3,868^{(3)}$ & $0,9970^{(4)}$ \\
\hline & \multicolumn{6}{|c|}{ Inclinação $22,85^{\circ}$} & \\
\hline Janeiro & 20,802 & 20,745 & $-0,056$ & 0,412 & $-0,270$ & 1,981 & 0,9975 \\
\hline Fevereiro & 19,726 & 19,997 & 0,271 & 0,405 & 1,375 & 2,055 & 0,9984 \\
\hline Março & 20,993 & 21,819 & $0,826^{(2)}$ & $1,170^{(2)}$ & $3,934^{(2)}$ & $5,573^{(2)}$ & 0,9843 \\
\hline Abril & 20,697 & 21,624 & 0,928 & 3,144 & 4,482 & 15,191 & 0,8839 \\
\hline Maio & 18,358 & 19,656 & $1,299^{(2)}$ & 1,426 & 7,074 & 7,765 & 0,9799 \\
\hline Junho & 17,863 & 19,229 & 1,366 & 1,553 & 7,646 & 8,692 & 0,9691 \\
\hline Julho & 18,658 & 20,073 & $1,415^{(2)}$ & 1,542 & 7,585 & 8,263 & 0,9598 \\
\hline Agosto & 18,827 & 18,905 & 0,079 & 2,362 & 0,417 & 12,546 & 0,9490 \\
\hline Setembro & 16,667 & 17,656 & 0,829 & 1,029 & 4,976 & 6,171 & 0,9944 \\
\hline Outubro & 20,306 & 20,610 & 0,304 & 0,701 & 1,496 & $3,452 b$ & 0,9958 \\
\hline Novembro & 21,807 & 21,777 & $-0,030$ & 0,304 & $-0,137$ & 1,392 & 0,9992 \\
\hline \multirow[t]{2}{*}{ Dezembro } & 19,925 & 19,624 & $-0,301$ & 0,420 & $-1,510$ & 2,110 & 0,9982 \\
\hline & \multicolumn{6}{|c|}{ Inclinação $32,85^{\circ}$} & \\
\hline Janeiro & 16,398 & 15,575 & $-0,823$ & 5,225 & $-5,019$ & 31,861 & 0,7531 \\
\hline Fevereiro & 17,856 & 18,132 & 0,332 & $3,114 \mathrm{a}$ & 1,861 & $17,439^{(2)}$ & 0,9142 \\
\hline Março & 19,166 & 20,439 & 1,273 & 2,130 & 6,640 & 11,115 & 0,9417 \\
\hline Abril & 20,500 & 21,235 & 0,736 & 1,126 & 3,588 & 5,492 & 0,9879 \\
\hline Maio & 17,019 & 19,344 & 2,326 & 5,475 & 13,665 & 32,171 & 0,7947 \\
\hline Junho & 19,267 & 20,994 & 1,727 & 2,029 & 8,964 & 10,529 & 0,9376 \\
\hline Julho & 19,003 & 20,891 & 1,888 & 2,147 & 9,934 & 11,299 & 0,9618 \\
\hline Agosto & 21,249 & 22,178 & 0,929 & 1,510 & 4,372 & 7,105 & 0,9509 \\
\hline Setembro & 20,538 & 21,729 & 1,191 & 1,285 & 5,799 & 6,258 & 0,9814 \\
\hline Outubro & 20,117 & 20,650 & $0,534^{(2)}$ & $0,947^{(2)}$ & $2,653^{(3)}$ & $4,706^{(3)}$ & $0,9849^{(4)}$ \\
\hline Novembro & 18,112 & 18,137 & 0,026 & 0,486 & 0,142 & 2,681 & 0,9969 \\
\hline Dezembro & 17,425 & 17,106 & $-0,320$ & 1,581 & $-1,834$ & 9,071 & 0,9661 \\
\hline
\end{tabular}

\section{Conclusões}

1. Quanto maior a transmissividade atmosférica do dia, maior a dispersão entre valores medidos e estimados de radiação global inclinada por modelos isotrópicos.
2. Menores ângulos de inclinação propiciam melhores desempenhos de modelos isotrópicos na estimativa da radiação global inclinada.

3. Os modelos de Liu \& Jordan, Jimenez \& Castro e Koronakis podem ser aplicados independentemente da condição de cobertura do céu. 
4. Em agrupamentos mensais, as equações de estimativa com base nas correlações entre os índices de claridade horizontal e inclinados possibilitam bons resultados.

\section{Referências}

AMARANTE, C.V.T. do; STEFFENS, C.A.; MOTA, C.S.; SANTOS, H.P. dos. Radiação, fotossíntese, rendimento e qualidade de frutos em macieiras 'Royal Gala' cobertas com telas antigranizo. Pesquisa Agropecuária Brasileira, v.42, p.925-931, 2007.

BORGES, V.P.; OLIVEIRA, A.S. de; COELHO FILHO, M.A.; SILVA, T.S.M. da; PAMPONET, B.M. Avaliação de modelos de estimativa da radiação solar incidente em Cruz das Almas, Bahia. Revista Brasileira de Engenharia Agrícola e Ambiental, v.14, p.74-80, 2010.

CENTRO DE PESQUISAS METEOROLÓGICAS E CLIMÁTICAS APLICADAS A AGRICULTURA. Clima dos municípios paulistas. Disponível em: <http://www.cpa. unicamp.br>. Acesso em: 30 jun. 2009.

CODATO, G.; OLIVEIRA, A.P.; SOARES, J.; ESCOBEDO, J.F.; GOMES, E.N.; PAI, A.D. Global and diffuse solar irradiances in urban and rural areas in southeast Brazil. Theoretical and Applied Climatology, v.93, p.57-73, 2008.

DE MIGUEL, A.; BILBAO, J.; DIEZ-MEDIAVILLA, M. Solar radiation incident on tilted surfaces in Burgos, Spain: isotropic models. Energy Conversion and Management, v.36, p.945951, 1995.

DIEZ-MEDiAVILLA, M.; DE MIGUEL, A.; BILBAO, J. Measurement and comparison of diffuse solar irradiance models on inclined surfaces in Valladolid (Spain). Energy Conversion and Management, v.46, p.2075-2092, 2005.

EL-SEBAII, A.A.; AL-HAZMI, F.S.; AL-GHAMDI, A.A.; YAGHMOUR, S.J. Global, direct and diffuse solar radiation on horizontal and tilted surfaces in Jeddah, Saudi Arabia. Applied Energy, v.87, p.568-576, 2010.

ESCOBEDO, J.F.; GOMES, E.N.; OLIVEIRA, A.P.; SORAES, J. Modeling hourly and daily fractions of UV, PAR and NIR to global solar radiation under various sky conditions at Botucatu, Brazil. Applied Energy, v.86, p.299-309, 2009.

EVSEEV, E.G.; KUDISH, A.I. The assessment of different models to predict the global solar radiation on a surface tilted to the south. Solar Energy, v.83, p.377-388, 2009.

GUEYMARD, C.A. Direct and indirect uncertainties in the prediction of tilted irradiance for solar engineering applications. Solar Energy, v.83, p.432-444, 2009.

IQBAL, M. An introduction to solar radiation. Toronto: Academic Press, 1983. 389p.

JIANG, Y. Estimation of monthly mean daily diffuse radiation in China. Applied Energy, v.86, p.1458-1464, 2009.
JIMENEZ, J.I.; CASTRO, Y. Solar radiation on sloping surfaces with different orientations in Granada, Spain. Solar Energy, v.28, p.257-262, 1982.

KAMALI, G.A.; MORADI, I.; KHALILI, A. Estimating solar radiation on tilted surfaces with various orientations: a study case in Karaj (Iran). Theoretical and Applied Climatology, v.84, p.235-241, 2006.

KORONAKIS, P.S. On the choice of the angle of tilt for south facing solar collectors in the Athens basin area. Solar Energy, v.36, p.217-225, 1986.

KUNZ, J.H.; BERGONCI, J.I.; BERGAMASCHI, H.; DALMAGO, G.A.; HECKLER, B.M.M.; COMIRAN, F. Uso da radiação solar pelo milho sob diferentes preparos do solo, espaçamento e disponibilidade hídrica. Pesquisa Agropecuária Brasileira, v.42, p.1511-1520, 2007.

LINDQUIST, J.L.; ARKEBAUER, T.J.; WALTERS, D.T.; CASSMAN, K.G.; DOBERMANN, A. Maize radiation use efficiency under optimal growth conditions. Agronomy Journal, v.97, p.72-78, 2005.

LIU, B.Y.H.; JORDAN, R.C. The interrelationship and characteristic distribution of direct, diffuse and total solar radiation. Solar Energy, v.4, p.1-19, 1960.

LOUTZENHISER, P.G.; MANZ, H.; FELSMANN, C.; STRACHAN, P.A.; FRANK, T.; MAXWELL, G.M. Empirical validation of models to compute solar irradiance on inclined surfaces for building energy simulation. Solar Energy, v.81, p.254-267, 2007.

NOORIAN, A.M.; MORADI, I.; KAMALI, G.A. Evaluation of 12 models to estimate hourly diffuse irradiation on inclined surfaces. Renewable Energy, v.33, p.1406-1412, 2008.

NOTTON, G.; CRISTOFARI, C.; POGGI, P. Performance evaluation of various hourly and daily slope irradiation models using Mediterranean experimental data of Ajaccio. Energy, Conversion and Management, v.47, p.147-173, 2006.

REVFEIM, K.J.A. A simple procedure for estimating global daily radiation on any surface. Journal of Applied Meteorology, v.17, p.1126-1131, 1978.

SCOLAR, J.; MARTINS, D.; ESCOBEDO, J.F. Estimativa da irradiação total sobre uma superfície inclinada a partir da irradiação global na horizontal. Revista Brasileira de Geofísica, v.21, p.249-258, 2003.

SOARES, J.; OLIVEIRA, A.P.; BOZNAR, M.Z.; MLAKAR, P.; ESCOBEDO, J.F.; MACHADO, A.J. Modeling hourly diffuse solar-radiation in the city of São Paulo using a neural-network technique. Applied Energy, v.79, p.201-204, 2004.

SOUZA, J.L. de; NICÁCIO, R.M.; MOURA, M.A.L. Global solar radiation measurements in Maceio, Brazil. Renewable Energy, v.30, 1203-1220, 2005.

SOUZA, P.J. de O.P. de; RIBEIRO, A.; ROCHA, E.J.P. da; FARIAS, J.R.B.; LOUREIRO, R.S.; BISPO, C.C.; SAMPAIO, L. Solar radiation use efficiency by soybean under field conditions in the Amazon region. Pesquisa Agropecuária Brasileira, v.44, p.1211-1218, 2009. 
TIAN, Y.Q.; DAVIES-COLLEY, R.J.; GONG, P.; THORROLD, B.W. Estimating solar radiation on slopes of arbitrary aspect. Agricultural and Forest Meteorology, v.109, p.67-74, 2001.

TIBA, C.; AGUIAR, R. de; FRAIDENRAICH, N. Analysis of a new relationship between monthly global irradiation and sunshine hours from a database of Brazil. Renewable Energy, v.30, p.957-966, 2005.

TURCO, J.E.P.; RIZZATTI, G.S. Avaliação de modelo matemático para estimar a radiação solar incidente sobre superfícies com diferentes exposições e declividades. Engenharia Agrícola, v.26, p.257-267, 2006.

Recebido em 1 de julho de 2009 e aprovado em 12 de março de 2010 